\title{
EFICACIA BIOLÓGICA DE HONGOS NEMATÓFAGOS PARA EL COMBATE DEL NEMATODO Globodera pallida (Stone) EN PAPA
}

\author{
Ricardo Piedra Naranjo, Miguel Obregón Gómez², Jorge Meckbel Campos³
}

\begin{abstract}
RESUMEN
El estudio se realizó en la localidad de San Juan de Chicuá provincia de Cartago, el lugar se localizó a 5 km del Volcán Irazú, con una altura de 2800 msnm, a 26 kilómetros al noreste de la provincia de Cartago. Predominó el bosque húmedo, el suelo era de origen volcánico del orden Andisol y la temperatura, precipitación y humedad relativa promedio anual fue de $15^{\circ} \mathrm{C}$, $2100 \mathrm{~mm}$ y $85 \%$, respectivamente. Se utilizó un diseño irrestricto al azar con 6 tratamientos y 4 repeticiones. Las cepas de hongos que se evaluaron fueron: Beauveria sp (JV), Paecilomyces lilacinus (CFI), Pochonia sp (Mog 08H), Lecanicillium lecanii, (BOS), Trichoderma sp (hongo nativo de la estación Carlos Durán) y un testigo absoluto. Se utilizaron $700 \mathrm{~g}$ de suelo esterilizado en cada tratamiento y una viabilidad infectiva promedio de 180 larvas y huevos por quiste. En la inoculación de quistes se usó una tela tipo muselina para determinar la eclosión de quistes y la recuperación del suelo al final de la investigación. El objetivo del estudio fue evaluar la eficacia de los microorganismos nematófagos. Los resultados mostraron diferencias estadísticas entre tratamientos, Trichoderma sp y Beauveria sp (JV), con respecto a los hongos Lecanicillium lecanii, Pochonia sp (BOS), Paecilomyces sp y el testigo absoluto en la variable de recuperación de quistes en el suelo. El tratamiento Trichoderma spp. fue el mejor, tanto en la no recuperación de quistes de suelo como un mayor peso de tubérculos de papa. Se debió validar y dar seguimiento a estos resultados obtenidos en condiciones de campo, los cuales son importantes como un elemento dentro de un manejo integrado de la plaga en las prácticas agronómicas del cultivo de papa en Costa Rica.
\end{abstract}

Palabras clave: Control biológico; viabilidad, eclosión, recuperación de quistes, eficacia.

1 ilnvestigador Nematólogo Instituto Nacional de Innovación y Transferencia de Tecnología Agropecuaria. 2231-5055. INTA. Costa Rica. Estudiante Doctorado en Ciencias Naturales Para el Desarrollo (DOCINAE).

2 Fitopatólogo, Profesor de Doctorado en Ciencia Naturales Para el Desarrollo (DOCINAE). San Francisco de Heredia. Costa Rica 8828-6382

3 Investigador Nematólogo Instituto Nacional de Innovación y Transferencia de Tecnología Agropecuaria. 2231-5055. INTA. Costa Rica 


\section{INTRODUCCION}

Entre los parásitos que atacan al cultivo de papa (Solanun tuberosum L.), los nematodos fitoparásitos fueron de gran importancia en muchos países del mundo. Setenta especies de nematodos se señalaron en el cultivo de la papa (ICTA 2002). Sin embargo, los formadores de quistes, Globodera rostochiensis (Woll) Behrens y G. pallida (Stone) Behrens, se consideraron como los más dañinos y afectaron el rendimiento de este cultivo (Greco 1992). Con el fin de realizar la investigación, fue necesario conocer el género y especie de Globodera spp para lo cual de utilizó la preparación de cortes perineales de los quistes y con el microscopio se realizó el conteo de las estrías cuniculares que se presentaron entre el ano y la vulva, Una forma de poder trabajar con Globodera spp y poder saber cual género y especie se iba a utilizar en la investigaciones fue, mediante la preparación de los cortes perineales de los quistes y a nivel de microscopio el conteo de las estrías cuniculares que se presentaron entre el ano y la vulva, G. rostochiensis poseía un promedio de 21,60 estrías y $G$. pallida 12,00 estrías (Greco 1993). El rango de huevos de cada quiste joven fue de 200-500 huevos, cuando se sembró el tubérculo de papa y creció el sistema radicular, estos produjeron exudados radicadles que estimuló la eclosión de los huevos, de los cuales emergieron los juveniles de segundo estadio (Greco 1992).

Las plagas agrícolas como Globodera spp se han tratado de controlar durante años mediante el empleo de plaguicidas químicos de fuerte impacto y negativos sobre los organismos benéficos que estaban presentes en el suelo, medio ambiente y ser humano, pero hoy día, se conoce que existen muchas actividades como el uso de hongos y organismos en el control de plagas en muchos cultivos como la papa (Crozzoli 1994). En la naturaleza, los hongos entomopatógenos puedieron ser eliminados o mantenidas las plagas bajo niveles que no ocasionaron daños económicos a los cultivos agrícolas (Azevedo, Melo 1998).
Una de las alternativas que existieron para reducir la utilización de nematicidas químicos fue utilizando organismos vivos para controlar los nematodos fitoparásitos (S. tuberosun). El manejo biológico de nematodos fitoparásitos es un componente esencial, tanto en el control integrado de plagas, como en la agricultura ecológica sostenible (Carballo y Guaharay 2004). El estudio del potencial de las bacterias y los hongos endofíticos para el control biológico de nematodos fitoparásitos fue adquiriendo gran importancia durante los últimos años, debido a la necesidad creciente de disminuir el uso de plaguicidas en los sistemas de producción agrícola (Marín 2003; Mena et al. 2003).

En el control de nematodos fitoparásitos, los hongos nematófagos fueron microorganismos con la capacidad de atacar, matar y digerir los nematodos (adultos, juveniles y huevos), aparte de su habilidad nematófaga muchos de esos hongos puedieron también vivir saprofíticamente en materia orgánica muerta, atacar a otros hongos (micoparásitos) y colonizaron raíces de plantas como endófitos. Hay más de 300 especies de hongos nematófagos que fueron descritos y encontrados por todo el mundo, incluyendo las regiones polares. Los hongos, habitantes del suelo, son generalmente más frecuentes en suelos con elevado contenido en materia orgánica. La mayoría de los nematodos fitopatógenos vivían en el suelo y atacaron las raíces de plantas, de manera que la posibilidad de usar hongos nematófagos para el control biológico de nematodos fitopatógenos fue utilizado e investigado en los últimos años con algún grado de importancia (Barron 2005).

Para la mayoría de los hongos endoparásitos se utilizaron sus esporas para infectar nematodos. Estos hongos fueron a menudo parásitos obligados de nematodos y fuera del cuerpo infectado del nematodo aparecieron sólo como estructuras de diseminación. Las esporas de estos hongos pudieron ser zoósporas móviles, como las de Catenaria spp. que se enquistaron sobre el nematodo adhiriéndose a él y penetrándole la cutícula 
con sus conidios adhesivos (www.ua.es/dpto/ dcmba/botanica/gefpv_inv1.html).

Conforme se conoció más de la ecología de los microorganismos con potencial para el combate biológico y sus relaciones con los patógenos y las plantas, el desarrollo comercial del combate biológico se incrementó aceleradamente (Arauz 1998).

De tal forma que el siguiente estudio tuvo como objetivo evaluar la eficacia hongos nematófagos contra el nematodo Globodera pallida (Stone). Analizando las variables de peso en $\mathrm{g}$ de cada tratamiento, número de quistes sin eclosionar, número de quistes eclosionados y cantidad de quistes recuperados en el suelo en cada tratamiento. Además, se observaron al microscopio de luz el parasitismo en los quistes tanto externa como internamente del mismo.

\section{MATERIALES Y MÉTODOS}

El estudio se desarrolló en San Juan de Chicuá de Oreamuno, provincia de Cartago, con una altitud de $2800 \mathrm{msnm}$, donde predominó el bosque muy húmedo montano, el suelo era de origen volcánico del orden Andisol y la temperatura, precipitación y humedad relativa promedio anual fueron de $15^{\circ} \mathrm{C}, 2100 \mathrm{~mm}$ y $85 \%$, respectivamente. El invernadero se ubicó a 90 56.9871 'latitud norte 83051.1316 longitud oeste en la misma localidad.

En la separación de quistes de suelo se utilizó el sistema de Fenwick (Fenwick 1940) modificado por Oostenbrink (Ostenbrink 1950). Este estaba conformado por un embudo colocado sobre una especie de jarra, el cuál en su parte ensanchada tenía un tamiz con poros de $1 \mathrm{~mm}$ de diámetro. El instrumento era de forma trapezoidal, en parte inferior se colocó un tamiz con poros de $1 \mathrm{~mm}$ de diámetro. El instrumento era de forma trapezoidal, y, en su parte inferior se encontraban los soportes del embudo y una aleta inclinada que bordeó la jarra como collar, pero que terminó en su solo conducto. La jarra tenía en su parte interior un tapón que se retiraba para desaguar y limpiar como se observó en la Figura 1.

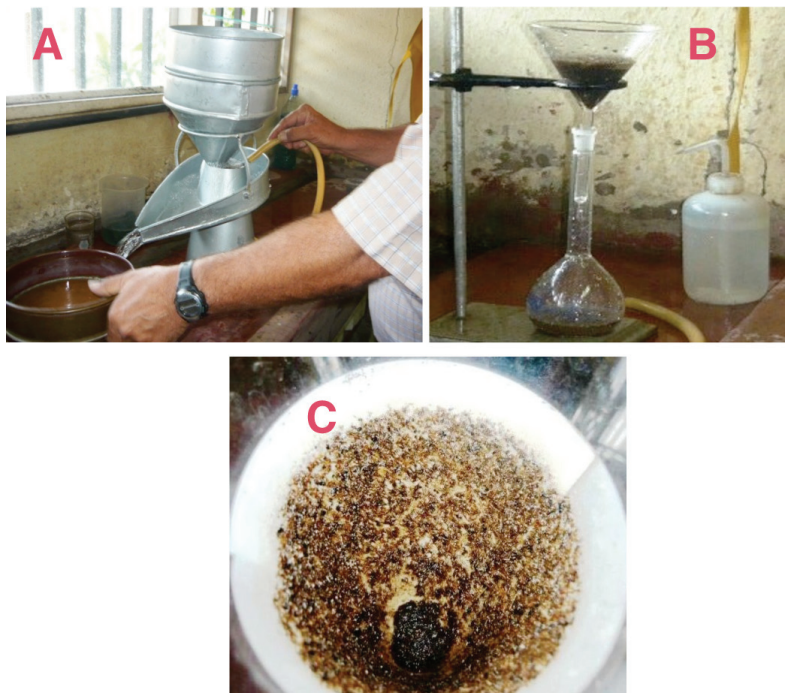

Figura 1. Fenwick modificado (A), balón aforado de $250 \mathrm{ml}$ (B) y filtro para la extracción de quistes (C). Fuente: Piedra Naranjo, R. San José, Costa Rica. 2007.

Los quistes recogidos en el tamiz de 100 mesh fueron transferidos a un balón aforado de 250 $\mathrm{ml}$ y llenado hasta la mitad con agua, se agitó y se mezcló la muestra y después se llenó el balón por completo con agua. Se dejó en reposo un minuto para que los quistes flotaran y el resto de materia orgánica precipitara, después se vaciaron los quistes sobre un papel filtro que fue colocado previamente en el embudo de manera que mientras se vaciara estuviera rotando sobre el balón y de esta manera se evitó que el material orgánico se pasara al filtro (Figura 1). La muestra se secó a temperatura ambiente de $24^{\circ} \mathrm{C}$ a $25^{\circ} \mathrm{C}$, posteriormente se realizó la extracción, selección y conteo de quistes.

Como se indicó en la literatura, un quiste tenía entre 200 hasta 500 o más huevos o larvas, pero en inoculaciones no se supo cuánto podía tener una población de quistes, es por tal razón que se realizó la prueba de viabilidad de los quistes (Figura 2). La viabilidad se hizo tomando 25 quistes, los cuales se trituraron con un homogenizador. Luego se disolvió en un volumen de agua de $50 \mathrm{cc}$. En una pipeta se tomó 3 cc y se obtuvo el promedio de huevos y larvas por quiste. Esta técnica se realizó como se indica a continuación: 
Fórmula de viabilidad de quistes

VT= Prom. 3cc xVol. $\mathrm{H}_{2} \mathrm{O}$

Donde:

$$
\begin{gathered}
\text { VT= Viabilidad Total } \\
\text { Prom }=\text { Promedio de } 3 \text { alícuotas } \\
Q=\text { Número de quistes }
\end{gathered}
$$

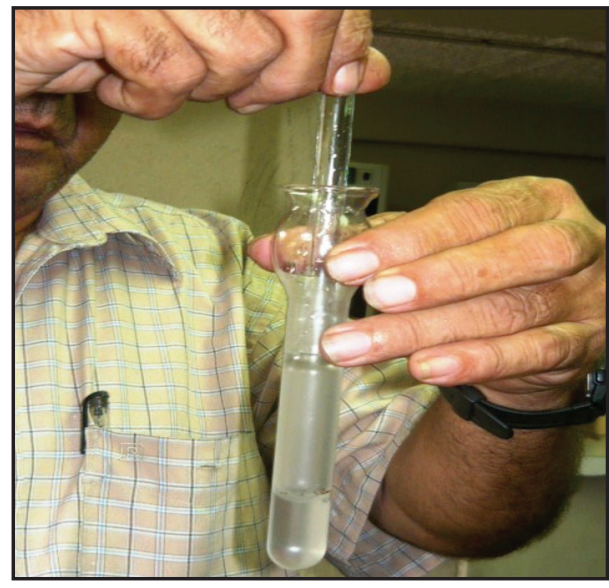

Figura 2. Homogenizador de quistes.

Una vez que se obtuvo la viabilidad de quistes para la inoculación, se realizó observaciones de cortes perineales y larvas al microscopio luz en 45x. Con esta acción se diagnosticó el género Globodera pallida (Stone), mismo que sirvió para la investigación en todos los tratamientos (Figura 3).

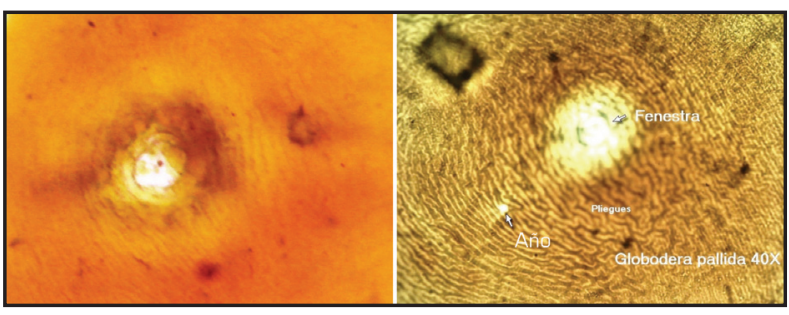

Figura 3. Identificacion de Globodera pallida mediante cortes perineales. Fuente: Naranjo Piedra, R. 2008.

Luego de la extracción de los quistes, se precedió a inocular y sembrar los tratamientos en macetas. Se utilizó un pote o maceta de $10 \times 10$ centímetros, se inocularon quistes sin eclosionar, número de quistes eclosionados, cantidad de quistes recuperados en el suelo en cada tratamiento y observación al estereoscopio, de quistes sin eclosionar recuperados de la malla. La cantidad de producto de cada hongo nematófago aplicado fue de $50 \mathrm{~g}$ en mezcla con arroz $80 \%$ de pureza. La concentración de los hongos fue de 1x109 .conidias por g de suelo Cuadro 1.

Cuadro 1. Tratamientos utilizadando cepas de hongos nativas de Costa Rica. San Juan de Chicuá. Cartago. 2008.

\begin{tabular}{lcccc}
\hline Tratamiento & $\begin{array}{c}\text { Concentración } \\
\text { (conidias/g) }\end{array}$ & $\begin{array}{c}\text { Código de } \\
\text { Cepas }\end{array}$ & $\begin{array}{c}\text { Producto } \\
\text { en g }\end{array}$ & $\begin{array}{c}\text { Quistes } \\
\text { por pote }\end{array}$ \\
\hline 1 Beauveria sp & $1 \times 109$ & $\mathrm{JV}$ & 50 & 50 \\
$\begin{array}{l}\text { 2.Paecilomyces } \\
\text { lilacinus }\end{array}$ & $1 \times 109$ & $\mathrm{CFI}$ & 50 & 50 \\
$\begin{array}{l}\text { 3.Pochonia sp } \\
\begin{array}{l}\text { 4.Lecanicillium } \\
\text { lecanii }\end{array}\end{array}$ & $1 \times 109$ & Mog 08H & 50 & 50 \\
$\begin{array}{l}\text { 5. Trichoderma sp } \\
\text { 6. } 1 \times 109\end{array}$ & BOS & 50 & 50 \\
\hline
\end{tabular}

Las cepas de los hongos nematófagos que se utilizaron eran nativas de diferentes zonas de Costa Rica. Se utilizó un diseño experimental irrestricto al azar con 6 tratamientos y 4 repeticiones (Cuadro 2). El análisis de varianza y la separación de medias de los tratamientos, se utilizó el software estadístico (Infostat 2008) con una prueba separación de medias Tukey alfa: 0,05.

Cuadro 2. Diseño experimental y distribución de los tratamientos. San José, Costa Rica. 2008.

\begin{tabular}{lllll}
\hline & \multicolumn{5}{c}{ Repeticiones } \\
\hline \multicolumn{1}{c}{ Tratamientos } & I & II & III & IV \\
\hline 1. Beauveria sp & 1 & 2 & 6 & 2 \\
2. Paecilomyces lilacinus & 3 & 4 & 4 & 4 \\
3. Pochonia sp & 4 & 5 & 5 & 5 \\
4. Lecanicillium lecanii & 5 & 3 & 3 & 3 \\
5. Trichoderma sp & 2 & 6 & 1 & 1 \\
6. Testigo absoluto & 6 & 1 & 2 & 6 \\
\hline
\end{tabular}




\section{RESULTADOS Y DISCUSIÓN}

Los resultados de esta investigación se evidenciaron en el Cuadro 3. La variable peso en g presentó diferencias estadísticas entre los tratamientos, lo que destacó a Trichoderma sp con un peso mucho mayor que los demás tratamientos, Figura 4. La variable quistes sin eclosionar, y quistes eclosionados no demostraron diferencias estadísticas entre los tratamientos. En la extracción y recuperación de quistes de suelo tanto Trichoderma sp como Beauveria sp, mostraron similitud en su análisis estadístico; pero diferentes con los demás tratamientos, siendo en esta variable los dos tratamientos mejores.

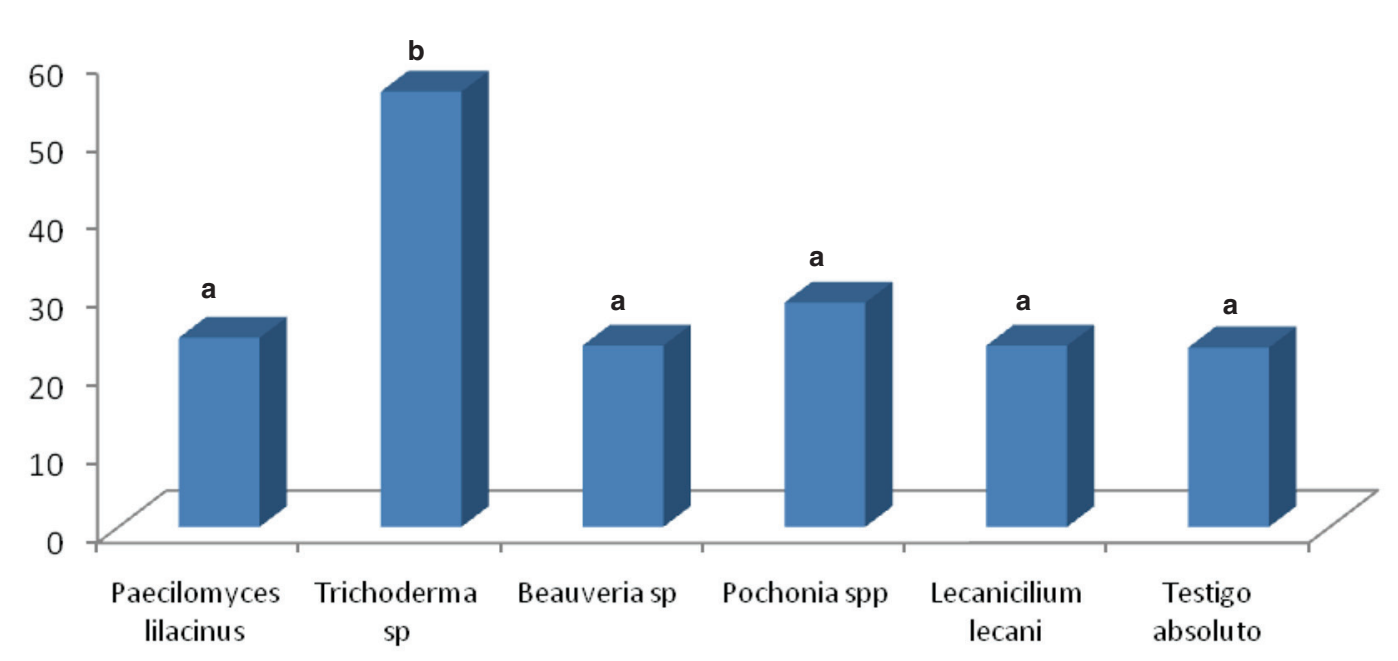

Figura 4. Efecto de los tratamientos con referencia al peso en g de tubérculos. Cartago. San Juan Chicuá. Cartago, Costa Rica. 2008.

Fue importante el análisis del testigo absoluto, ya que los quistes inoculados, eclosionaron de forma normal con los exudados radicales de las plantas, además, existió la presencia de humedad en la maceta. La eclosión de quistes y la formación de los mismos en raíces se pudo verificar cuando se hizo la extracción y recuperación de quistes del suelo al final de la investigación y este análisis reflejó una gran diferencia con lo demás tratamientos en su análisis estadístico.

En forma general, tanto en la variable de peso en g como en la extracción y recuperación de quistes del suelo el tratamiento Trichoderma sp, presentó un mejor resultado, Cuadro 3 y Figura 4.

Cuadro. 3. Resultados y separación de medias de los tratamientos de hongos nematófagos. San Juan de Chicuá. Cartago, Costa Rica. 2008.

\begin{tabular}{lcccc}
\hline Tratamientos & Peso en g & $\begin{array}{c}\text { Quistes sin } \\
\text { eclosionar }\end{array}$ & $\begin{array}{c}\text { Quistes } \\
\text { eclosionados }\end{array}$ & $\begin{array}{c}\text { Quistes } \\
\text { reuperados }\end{array}$ \\
\hline Paecilomyces lilacinus & $24,25 \mathrm{a}$ & $24,5 \mathrm{a}$ & $25,50 \mathrm{a}$ & $2,25 \mathrm{ab}$ \\
Trichoderma sp & $\mathbf{5 5 , 7 5} \mathbf{b}$ & $27,75 \mathrm{a}$ & $22,25 \mathrm{a}$ & $\mathbf{0 , 0} \mathbf{~ a}$ \\
Beauveria sp & $23,25 \mathrm{a}$ & $31,00 \mathrm{a}$ & $19,00 \mathrm{a}$ & $\mathbf{0 , 2 5} \mathbf{a}$ \\
Pochonia spp & $28,75 \mathrm{a}$ & $33,75 \mathrm{a}$ & $16,25 \mathrm{a}$ & $7,75 \mathrm{ab}$ \\
Lecanicilium lecani & $23,25 \mathrm{a}$ & $35,00 \mathrm{a}$ & $15,00 \mathrm{a}$ & $12,00 \mathrm{~b}$ \\
Testigo absoluto & $23,00 \mathrm{a}$ & $36,00 \mathrm{a}$ & $14,00 \mathrm{a}$ & $25,00 \mathrm{c}$ \\
\hline
\end{tabular}

Letras distintas entre columnas indicaron diferencias significativas según Tukey $(p<=0,05)$ 
En la Figura 5 y cuando se analizó un poco más el resultado de la recuperación de quistes fue quizá la prueba más notoria del efecto sobre todo cuando se constató que el hongo Trichoderma sp, el cual se inoculó, funcionó y éste cuando se comparó con el testigo absoluto, mostró una gran diferencia. Por otro lado con respecto al tratamiento Beauveria $s p$ hubo que destacar que al haber encontrado semejanzas con el tratamiento Trichoderma sp. pudo ser un tratamiento promisorio en otras investigaciones; sin embargo, el mismo tratamiento no evidenció datos importantes en el peso de los tubérculos por lo que tuvo un comportamiento parecido a los demás.

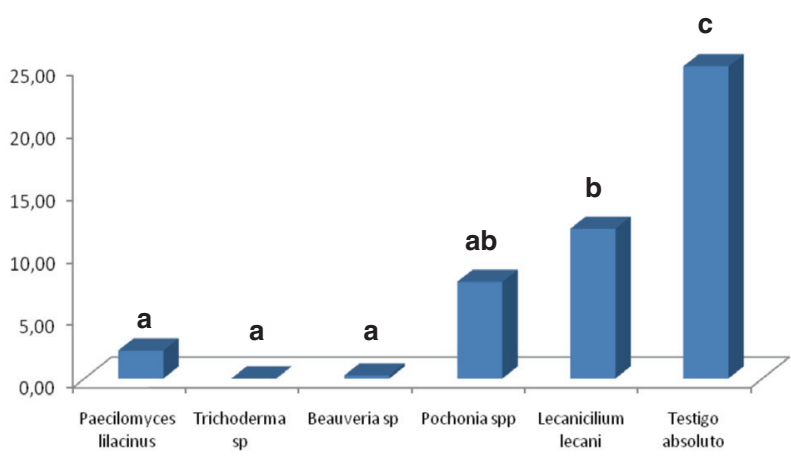

Figura 5. Efecto de tratamientos en la recuperación de quistes (Globodera pallida Stone) de suelo. Cartago. San Juan de Chicuá. Cartago, Costa Rica. 2008.

Los quistes recuperados de la malla (misma que se usó para inocular los tratamientos) de la cepa de Trichoderma sp, se realizó observación de quistes al estereoscopio de luz, es decir los quistes que no eclosionaron. Exceptuando Trichoderma sp, los demás tratamientos no se observó algún hongo o enemigo natural que afectó a los quistes. En el tratamiento con Trichoderma sp. se pudo observar al microscopio de luz, por medio del cual se tomaron fotografías que demostraron los síntomas de micelio del hongo tanto en el quiste como en larvas y huevos, Figura 6.
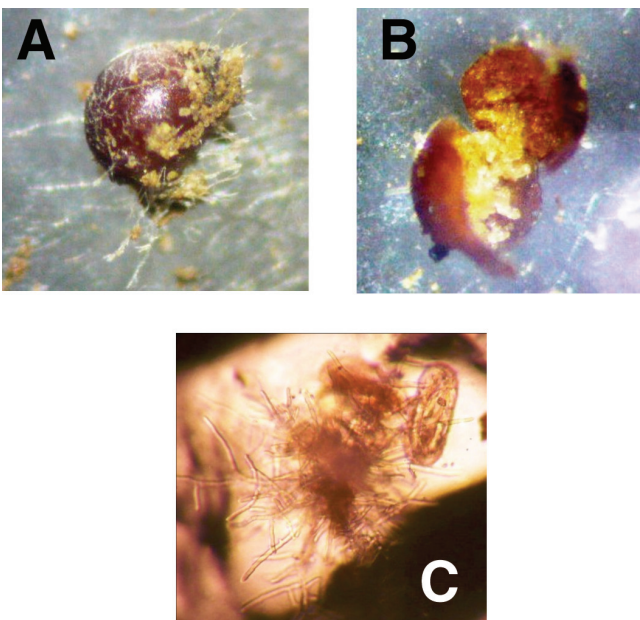

Figura 6. Quiste afectados con hongo (A), huevos desechos y afectados dentro de quistes (B) y huevos afectados con hongo (C), todo observado en tratamiento Trichoderma sp. Fuente: Piedra Naranjo, R. San José, Costa Rica. 2008.

También se observó micelio en la parte externa del quiste y dentro del mismo, conidias de Trichoderma sp. Se aislaron algunos quistes en medio de cultivo PDA y este demostró crecimiento en el medio de cultivo, lo que evidenció que el hongo Trichoderma sp penetró al quiste e invadió afectando larvas y huevo, Figura 7.
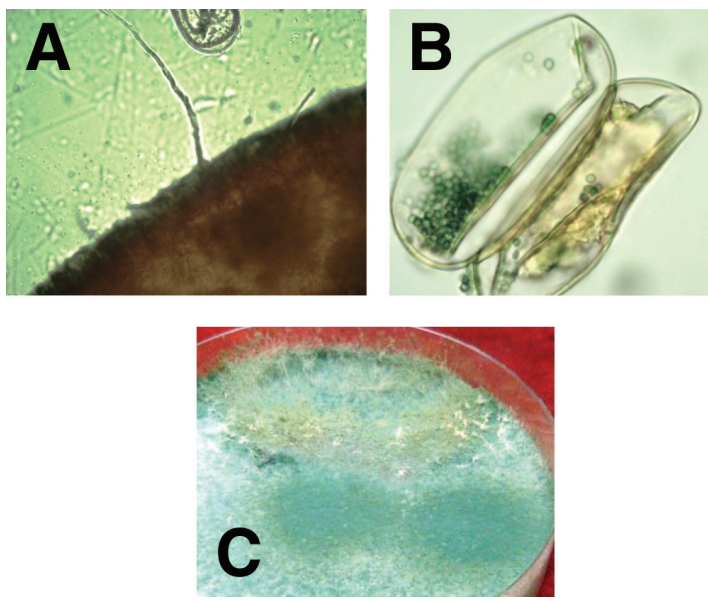

Figura 7. Parte externa de quiste con micelio ( A), conidias de Trichoderma sp, dentro huevos (B) y crecimiento de Trichoderma sp en medio de cultivo PDA (C). Fuente: Piedra Naranjo, R. San José, Costa Rica. 2008. 


\section{CONCLUSIONES}

El efecto de los mecanismos de acción en la cepa de Trichoderma spp como agente biológico quedó demostrado en esta investigación contra Globodera pallida (Stone). Los resultados de la eficacia y control del nematodo de quiste $G$. pallida fue demostrado con análisis estadísticos, recuperación de quistes del suelo, peso en g de tubérculos de papa y observaciones al estereoscopio de luz los quistes atacados por Trichoderma sp.

La acción de Trichoderma $s p$ favoreció el desarrollo del sistema radicular de la planta, esto lo demostró la mayor cantidad de peso en los tubérculos de papa que indudablemente indujo a que la planta aumentara la producción.

El efecto del hongo Beauveria $s p$ en la recuperación de quistes del suelo dio diferencias estadísticas muy semejantes al tratamiento Trichoderma $s p$, pero no así en el peso en gramos de tubérculos de papa. Sin embargo; esta cepa de hongo no se debió descartar y seguir investigando debido a que es un microorganismo con historial de nematófago en otros géneros de nematodos fitoparásitos.

Es importante evaluar la cepa Trichoderma sp (Carlos Durán) contra otros patógenos como Rhizoctonia solani, esto por ser un patógeno de importancia en la mayoría de fincas productoras de papa y que está muy relacionada con nematodos formadores de quistes como G. pallida. De igual forma investigar esta cepa de Trichoderma sp, y caracterizar molecularmente su especie y valorar entre otras aspectos su compatibilidad con productos químicos como nematicidas y fungicidas utilizados en el cultivo de papa, en especial la zona norte de Cartago.

Los resultados son importantes para considerar el manejo integrado de esta plaga y en prácticas agronómicas en fincas productoras de papa en Costa Rica.
Se debió dar un seguimiento y validar las cepas de Trichoderma spp. y Beauveria sp. bajo condiciones de campo. Entre otras aspectos, estos hongos debieron tener una prueba de calidad antes de ser aplicados y así poder tener certeza que indique la concentración de conidias o esporas de los hongos por gramos de suelo.

\section{LITERATURA CITADA}

Arauz, I.F. 1998. Fitopatología: Un enfoque agroecológico. San José, Costa Rica. Editorial de la Universidad de Costa Rica. P 467.

Azevedo, J.L; Melo, I.S. (1998) Control microbiano de insectos - plagas en mejoramento genético. Controle Biológico 1: 69-93

Barron, G.L. 1977. Actualizado 2005. The NematodeDestroying Fungi. Topics In Mycobiology No.1. Canadian Biological Publications Ltd., Guelph. Sp.

Carballo, M.; Guaharay, F. 2004. Control biológico de plagas agrícolas. Serie Técnica manual técnico . Managua: Centro Agronómico Internacional de Agricultura Tropical y Enseñanza (CATIE) No. 53. pp-186-199.

Cozzoli. P.; Renato. 1994. Temas de nematología Agrícola I. Universidad Central de Venezuela, Facultad de Agronomía, Comisión de información pp. 8-9.

InfoStat. 2008. InfoStat, versión 2008. Manual del Usuario. Grupo InfoStat, FCA, Universidad Nacional de Córdoba. Primera Edición, Editorial Brujas Argentina.

Greco, N.; Moreno, I.L. 1992 Influence of Globodera rostochiensis on yield of summer, winter and spring sowed potato in Chile. Nematropica 22:165-173.

Greco, N.; D’addabbo, T.; Brandonisio, A.; Elia, F. 1993. Damage to Italian crops caused by cyst-forming nematodes. J. Nematol. 25(4S):836-842.

Marín, D.H. 2003. Research in progress and future perspectives on the root system management (abstract). In Turner, DW; Rosales, FE. eds. Banana root system: towards a better understanding for its productive management. Proceedings of an international symposium held in San José, Costa Rica, 3-5 November 2003. National Banana Corporation (CORBANA). p. 23. 